\title{
An Optimal Receiver for CDMA forward link without pilot tone*
}

\author{
Piero Castoldi ${ }^{\dagger}$ and Hisashi Kobayashi \\ Department of Electrical Engineering \\ School of Engineering and Applied Science \\ Princeton University \\ Princeton, NJ 08544, USA \\ Tel: 609258 1984, Fax: 6092583745 \\ E-mail: hisashi@ee.princeton.edu
}

\begin{abstract}
We derive an optimal receiver for the forward link of a CDMA mobile radio system subject to slowly varying Rayleigh fading. The proposed optimal detector does not require the transmission of a pilot tone and exploits fading memory and Kalman filtering for channel estimation. The receiver exhibits good performance especially for low signal-to-noise ratio which is the typical operating conditions of CDMA systems.
\end{abstract}

\section{Introduction}

The North American digital cellular standard IS-95 employs Code Division Multiple Access (CDMA) based on direct sequence spread spectrum (DS/SS) $[1,2]$. The most significant impairment that a forward link (a channel from the base station to mobile receivers) suffers is the effect of Rayleigh fading (flat or frequency selective). The traditional approach to handle this problem is to use a pilot tone which is basically a DC component in the baseband and is superimposed to signals to be sent to mobile receivers [1]-[3]. The pilot tone enables each mobile receiver to estimate its channel (especially the effect of fading) and perform coherent detection. But the insertion of the pilot tone costs the system a certain amount of power assigned to that purpose. For example, in the IS-95 CDMA, the pilot signal is approximately $6 \mathrm{~dB}$ above the signal level of each data channel.

In this paper we consider a system without such a pilot tone, and derive an optimal receiver for the forward link, when the received signal is affected by Rayleigh fading. For the actual implementation of the receiver a persurvivor Kalman processing technique [6] is adopted. The paper shows how this technique can be easily applied to single-user receivers employed in mobile terminals of a CDMA system.

${ }^{*}$ This work has been supported, in part by the DOD/FRI, Arpa/Hanscom, the National Science Foundation and the Ogasawara Foundation for the Promotion of Science and Engineering.

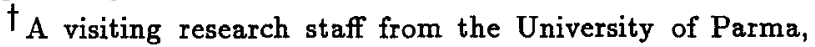
sponsored by Consiglio Nazionale delle Ricerche (CNR), Italy

0-7803-3905-3/97/\$10.00 ${ }^{\circledR} 1997$ IEEE
Some limited amount of pilot-like signal may be required to periodically train the receiver, but there will be no need for a permanent injection of the pilot signal. Alternatively known pilot symbols, which do not cause any power overhead, have to be inserted in order to train the receiver.

\section{System model}

We denote the information sequence $\ddagger$ (whose length is $M$ ) destined to the $k$-th user by $\mathrm{x}^{(k)}=\left\{x_{m}^{(k)}\right\}_{m=1}^{M}$, $k=1,2, \ldots, K$; the $k$-th user's signature by $s^{(k)}(t)$, which is a function supported only over the bit interval $[0, T]$, where $T$ is the bit interval. The $k$-th user signature

$$
s^{(k)}(t)=\sum_{n=1}^{G} b_{n}^{(k)} g\left(t-n T_{c}\right)
$$

is a unit-energy, binary PAM signal based on the Hadamard sequence (or called the Hadamard-Walsh function) $\mathbf{b}^{(k)}=\left\{b_{i}^{(k)}\right\}_{i=1}^{G}$, where $G$ is the length of the Hadamard sequence ( $G=64$ in the IS-95) and is also called the processing gain of the CDMA system; $g(t)$ is a rectangular pulse of duration $T_{c}=\frac{T}{G}$. The signatures $s^{(k)}(t)$ of the $K$ users to be served by the base station are mutually orthogonal, and are used to modulate every bit of the data $\mathrm{x}^{(k)}$.

Then, assuming a linear modulation (e.g., QAM or PSK) we can express the transmitted signal in the forward link in the following baseband form:

$$
s(t)=\sum_{m=1}^{M} \sum_{k=1}^{K} x_{m}^{(k)} s^{(k)}(t-m T)
$$

In the IS-95 system the above baseband signal is multiplied by a pseudorandom sequence (an M-sequence of

\footnotetext{
'If the modulation is QAM or QPSK, as in the IS-95, the information sequence is complexed valued: the real and imaginary components represent sequences that should be fed into the in-phase and quadrature-phase components of the modulator.
} 
very "long" period) to scramble the data. We drop this multiplicative term, since we assume that each receiver descrambles the received signal immediately after demodulation, hence the effect of the scrambling/descrambling is canceled out, and additive white Gaussian noise (AWGN) will retain its statistical property.

The fading is supposed to be frequency selective and Rayleigh distributed. The received signal at the $k$-th mobile user can be written as:

$$
r(t)=\sum_{m=1}^{M} \sum_{k=1}^{K} x_{m}^{(k)} \sum_{l=0}^{L-1} f_{m, l} s^{(k)}\left(t-m T-l T_{c}\right)+w(t)
$$

where $f_{m, l}$ represents the $l$-th echo's slowly changing signal amplitude at epoch $m$, and can be assumed constant during a bit interval. The $L$ time-varying fading coeffcients $\left\{f_{m, l}\right\}_{l=0}^{L-1}$ are supposed to be correlated in time (subscript $m$ ), but independent from path to path. The multipath delays are supposed to be integer multiples of the chip interval $T_{c}$.

\section{Optimal detection}

We derive an optimal receiver structure based on the maximum likelihood sequence estimation (MLSE) criterion. It is rather straightforward to extend the MLSE receiver that is described in [4] for a class of linear modulation. The problem can be reformulated as a joint parameter estimation problems where the estimated parameters include data sequence $\mathrm{x}^{(k)}$ and fading coefficients $f_{m, l},(l=0,1, \ldots, L-1 ; m=1,2, \ldots, M)$.

The preliminar front-end processing in order to extract a sufficient statistics from the received signal is realized using a bank of filters matched to the $k$-th users signature with proper sampling [4]. The output of each filter is sampled at bit rate. At the $m$-th signaling interval, the observation $y_{m, l}^{(k)}$ of the $k$-th user, regarding the $l$-th multipath echo is given by:

$$
\begin{gathered}
y_{m, l}^{(k)}=\int_{m T}^{(m+1) T} r(t) s^{(k)}\left(t-m T-l T_{c}\right) d t= \\
=x_{m}^{(k)} f_{m, l}+\sum_{\substack{i=1, n=0 \\
(i, n) \neq(k, l)}}^{K, L-1} x_{m}^{(i)} f_{m, n} \rho_{(i, n)(k, l)}+w_{m, l}^{(k)} \\
k=1,2, \ldots, K \quad l=0,1, \ldots, L-1
\end{gathered}
$$

where

$$
\rho_{(i, n)(k, l)}=\int_{0}^{T} s^{(i)}\left(t-n T_{c}\right) s^{(k)}\left(t-l T_{c}\right) d t
$$

is the cross-correlation between the $i$-th users signature delayed according to the $n$-th path lag and that of the $k$-th user delayed according to the $l$-th path lag, and

$$
w_{m, l}^{(k)}=\int_{m T}^{(m+1) T} s^{(k)}\left(t-m T-l T_{c}\right) w(t) d t
$$

Let us observe that (4) is further simplified when the fading is flat $(L=1)$, because the multiple access interference (MAI) disappears:

$$
y_{m, l}^{(k)}=x_{m}^{(k)} f_{m, l}+w_{m, l}^{(k)}
$$

Equation (4) strictly holds when the fading is flat $(L=1$, we can drop the index $l$ ) or, approximately when $L>1$ and MAI mitigation technique are employed, so that the second term in (4) is negligible.

Then, detection based on MLSE is accomplished using the observations (7). In order to simplify the notation we drop the identifier $k$ and define the following vectors

$$
\begin{aligned}
\mathbf{y}_{m, l} & =\left(y_{1, l}, y_{2, l}, \ldots, y_{m, l}\right) \\
l & =0,1, \ldots, L-1 \\
\mathbf{Y}_{m} & =\left(\mathbf{y}_{1}, \mathbf{y}_{2}, \ldots, \mathbf{y}_{m}\right) \\
\mathbf{x}_{m} & =\left(x_{1}, x_{2}, \ldots, x_{m}\right)
\end{aligned}
$$

The MLSE criterion requires to perform the following maximization (see e.g. [4]) in order to extract the ML sequence $\hat{\mathbf{x}}_{M}$ of length $M$ :

$$
\hat{\mathbf{x}}_{M}=\arg \max _{\mathbf{x}_{M}} p\left(\mathbf{Y}_{M} \mid \mathbf{x}_{M}\right)
$$

In order to perform the above maximization we need to express in closed form the joint probability density function $p\left(\mathbf{Y}_{M} \mid \mathbf{x}_{M}\right)$. For actual detection we need to formulate a fixed complexity recursive algorithm, for the maximization problem in (11). In [5] a recursive formulation which holds regardless of the fading distribution is presented. The probability density function in (11) can indeed be expressed in closed form since both the fading and the noise are Gaussian distributed. However some assumptions have to be introduced to derive a recursive formulation.

Here, in order to simplify the above maximization we assume that the fading correlation can be modeled by an autoregressive moving average (ARMA) model. It can be easily shown, using Kalman filter theory, that the above maximization leads to the minimization of the following likelihood function $[5,6]$

$$
\begin{aligned}
\Lambda\left(\mathbf{x}_{M}\right) & =\sum_{m=1}^{M} \sum_{l=1}^{L}\left\{\frac{\left|y_{m, l}-\overline{y_{m \mid m-1, l}}\right|^{2}}{\sigma_{m \mid m-1, l}^{2}}\right. \\
& \left.+\log \sigma_{m \mid m-1, l}^{2}\right\}
\end{aligned}
$$

in which

$$
\begin{array}{r}
\overline{y_{m \mid m-1, l}}=E\left[y_{m, l} \mid \mathbf{y}_{m-1, l}, \mathbf{x}_{m}\right] \\
\sigma_{m \mid m-1, l}^{2}=E\left[\left|y_{m, l}-\overline{y_{m \mid m-1, l}}\right|^{2} \mid \mathbf{y}_{m-1, l}, \mathbf{x}_{m}\right]
\end{array}
$$

are, respectively, the conditional mean and variance of $y_{m, l}$ given $\mathbf{y}_{m-1, l}$ and $\mathbf{x}_{m}$ for $l=0,1, \ldots, L-1$. These 
quantities depends on $x_{m}$ and are computed recursively utilizing the well known technique of per-survivor Kalman processing [6,7].

We refer to the following $(1,0)$-order ARMA model to describe the fading evolution:

$$
f_{m, l}=F \cdot f_{m-1, l}+G \cdot v_{m, l} \quad l=0,1, \ldots, L-1
$$

where $F$ and $G$ are evaluated from the second order statistics of the channel, as shown in Appendix.

The conditional mean $\overline{y_{m \mid m-1, l}}$ and the conditional variance $\sigma_{m \mid m-1, l}^{2}(l=0,1, \ldots, L-1)$ in (12) are evaluated according to [6]:

$$
\begin{aligned}
& \overline{y_{m \mid m-1, l}}=x_{m} \hat{f}_{m \mid m-1, l} \\
& \sigma_{m \mid m-1, l}^{2}=\left|x_{m}\right|^{2} \Sigma_{m \mid m-1, l}+\sigma_{w}^{2}
\end{aligned}
$$

where $\sigma_{w}^{2}$ is the noise variance in (4), and $\hat{f}_{m \mid m-1, l}$ and $\Sigma_{m \mid m-1, l}(l=0,1, \ldots, L-1)$ are, respectively, conditional mean and variance of the fading coefficients given its past values. $\hat{f}_{m \mid m-1, l}$ and $\Sigma_{m \mid m-1, l}$ are determined by the following classical scalar recursions

$$
\begin{aligned}
k_{m, l} & =F \Sigma_{m \mid m-1, l} x_{m} / \sigma_{m \mid m-1, l}^{2} \\
\Sigma_{m+1 \mid m, l} & =\left(F-k_{m, l} x_{m}\right) \Sigma_{m \mid m-1, l} F^{*}+|G|^{2} \\
\hat{f}_{m+1 \mid m, l} & =\left(F-k_{m, l} x_{m}\right) \hat{f}_{m \mid m-1, l}+k_{m, l} y_{m, l} \\
l & =0,1, \ldots, L-1
\end{aligned}
$$

with initial conditions:

$$
\begin{aligned}
\hat{f}_{0 \mid-1, l} & =E\left[f_{m, l}\right]=0 \\
\Sigma_{0 \mid-1, l} & =E\left[f_{m, l} f_{m, l}^{*}\right] \\
l & =0,1, \ldots, L-1
\end{aligned}
$$

\section{Numerical results}

In Fig. 1 we report the numerical results obtained by computer simulation for the proposed receivers. The performance measure is the average Bit Error Rate (BER) versus $E_{b} / N_{0}$ where $E_{b}$ is the received signal energy per bit and $N_{0}$ is the noise spectral density. More specifically, a performance comparison between the proposed optimal detector and the coherent detector which makes use of a pilot tone [3] is presented. As in [3], a BPSK modulation format is employed and a spreading factor $G=64$ is considered. The pilot tone amplitude of the coherent detector, used as performance reference, is $A=6 \mathrm{~dB}$.

The Rayleigh fading is supposed to be flat (hence $L=$ 1). A (1,0)-order ARMA channel model (completely specified by a parameter $\rho$ ) is assumed to account for the fading correlation. The smaller the parameter $\rho(0<\rho<1)$ the faster the channel variations. The considered values of $\rho=0.99999,0.9999,0.999$ correspond to the correlation between symbol-spaced fading samples when the normalized the Doppler band $B_{d}=f_{d} T=0.001,0.003,0.01$

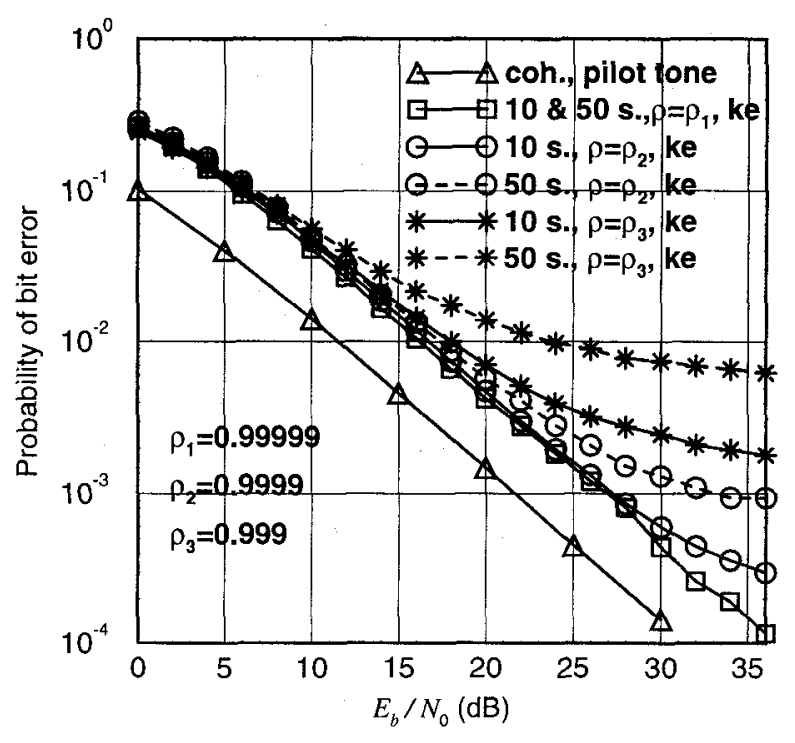

Figure 1: Symbol error rate versus $E_{b} / N_{0}$.

where $f_{d}$ is the maximum Doppler frequency. The coherent detector, used as a performance reference, is almost not sensitive to the Doppler band $B_{d}$ for the considered values, hence a unique performance curve marked with triangles is shown.

The proposed detector requires a periodic training of the Kalman filters utilizing a known symbol. The training method is based on the periodic insertion of a known symbol in the data stream (data-trained receiver). Two training intervals are considered in the numerical results: in one case the receiver is retrained every 10 information symbols and in the other case every 50 information symbols.

The coherent detector and the Kalman receiver which exhibits the best performance $\left(\rho=\rho_{1}\right)$ are separated by approximately $6 \mathrm{~dB}$ at low signal-to-noise ratio and approximately $5 \mathrm{~dB}$ for high signal-to-noise ratio. Furthermore, for $\rho=\rho_{1}$ the Kalman receiver does not exhibit an appreciable difference in performance adopting any of the two training rates, hence a unique curve marked with squares is reported. If we consider that the pilot tone requires an overhead of $6 \mathrm{~dB}$ of transmitting power, the performance of the proposed detector for $\rho=\rho_{1}$ is equivalent, in terms of power efficiency, to that of the coherent detector for low signal to-noise-ratio and better for high-signal-to noise ratio. On more slowly-varying channels the performance (not reported) is even better. For faster varying channel the proposed receiver structure is not attractive for high signal to-noise-ratio because an error floor appears and the performance degrades severely. For low signal-to-noise ratio the performance is still acceptable.

The most interesting feature of the Kalman detector is that the post processing after the matched filter (i.e. the Kalman filter iterations for channel estimation) is done 
at symbol rate. On the other hand, channel estimation in the coherent detector requires a chip rate sampler in order to average out the effect of noise [3].

There is an alternative way to train the proposed receiver. It consists in providing the Kalman filter with an initial condition which is close to the true channel estimate. This can be done by periodic insertion, of a pilot tone for a symbol interval duration, which can be used to obtain a good channel estimate. This channel estimate can be used in (22) instead of the initial zero value. No pilot symbols are needed in this case, and the performance of the receiver (not reported) adopting the same training intervals ( 10 or 50 symbols) are slightly superior to that of the data-trained receiver. A drawback of this training mode is that some power has to be injected periodically to transmit this pilot tone.

\section{Conclusions}

We have shown an application of the per-survivor Kalman processing to a single-user receiver for the forward link of a radio CDMA system. Multiuser detection, though conceivable, is not usually employed at the mobile receiver side, because of its heavy computational complexity. The proposed receiver and the traditional coherent receiver used in the forward link of the IS-95 system has equivalent performance up to moderate signal-tonoise ratios and when the channel is slowly varying. As a consequence the proposed detector may qualify as a competitor with the advantage that the Kalman processing is performed at symbol rate, while the coherent detector needs a chip-rate processing in order to obtain a channel estimate. Still for moderately-varying channel, though the performance of the proposed detector is slightly degraded, trade-offs between performance and complexity may be realized.

\section{Appendix}

We clarify the aspect of approximating the correlation of one fading coefficient $f_{m, l}$ with an arbitrary $(M, N)$-order ARMA model. If we assume the Doppler shift of $f_{d}[\mathrm{~Hz}]$, then the power spectral density of the fading process $f_{l}(t)$ is given by

$$
P(f)=\frac{\sigma^{2}}{\pi f_{d} \sqrt{1-\left(\frac{f}{f_{d}}\right)^{2}}}
$$

where $\sigma^{2}$ is the power of the fading process and its autocorrelation function $\Phi(\tau)$ is

$$
\Phi(\tau)=\sigma^{2} J_{0}\left(2 \pi f_{d} \tau\right),
$$

where $J_{0}(x)$ is the Bessel function of order zero. Then the sampled fading amplitude sequence $f_{m}$ has the discrete normalized autocorrelation function

$$
R_{l}[j]=E\left[f_{m, l} f_{m+j, l}^{*}\right]=J_{0}\left(2 \pi f_{d} j T_{b}\right),
$$

where $T_{b}$ is the bit interval, i.e., $T_{b}=G T_{c}$.

From the knowledge of $R_{l}[j], j=1,2, \ldots$ we can approximate the above fading correlation with an $(M, N)$ ARMA model [8]. If we define the fading vector

$$
\mathbf{f}_{m-1, l}=\left(f_{m-1, l}, f_{m-2, l}, \ldots, f_{m-N, l}\right),
$$

and the innovations vector (i.i.d. Gaussian random variable with zero mean and unit variance)

$$
\mathbf{v}_{m, l}=\left(v_{m, l}, v_{m-1, l}, \ldots, v_{m-M, l}\right)
$$

the $(M, N)$-order ARMA model is expressed as follows:

$$
f_{m, l}=\mathbf{F} \cdot \mathbf{f}_{m-1, l}+\mathbf{G} \cdot \mathbf{v}_{m, l}
$$

and unit variance. The time-invariant vectors $\mathbf{F}$ and $\mathbf{G}$ are determined by ARMA identification techniques [8]. The model in (15) is a $(1,0)$-order ARMA model so that $\mathbf{F}$ and $\mathbf{G}$ are numbers.

\section{References}

[1] A. Salmasi, K. S. Gilhousen, "On the System Design Aspects of Code Division Multiple Access (CDMA) applied to Digital Cellular and Personal Communications Networks", Proc. of Vehicular Technology Conference 1991.

[2] A.J. Viterbi, "CDMA, principles of spread spectrum communication", Addison-Wesley, 1995

[3] P. Castoldi, A. Yoshimura and H. Kobayashi, "Performance bounds for adaptive detection in mobile Radio Systems", Conference on Information Science and Systems '97, Johns Hopkins University, Baltimore MD, March 1997.

[4] H. Kobayashi, "Simultaneous Adaptive Estimation and Decision Algorithm for Carrier Modulated Data Transmission Systems", IEEE Trans. on Commun. Technology, Vol. COM-19, No. 3, June 1971, pp. 268280.

[5] P. Castoldi, R. Raheli, "Recursive Optimal Detection of Linear Modulations in the Presence of Random Fading", submitted to European Transactions on Telecommunications and related Technologies.

[6] Q. Dai, E. Shwedyk, "Detection of Bandlimited Signals Over Frequency Selective Rayleigh fading Channels", IEEE Trans. on Commun., vol. 42, Feb./Mar./Apr. 1994.

[7] R. Raheli, A. Polydoros, C.-K. Tzou, "Per-Survivor Processing: A General Approach to MLSE in Uncertain Environments," IEEE Trans. Commun., vol. COM-43, pp. 354-364, Feb.-Apr. 1995.

[8] A. Papoulis, "Probability, Random variables and stochastic processes", 3rd edition, McGraw Hill, 1991. 